TRANSACTIONS OF THE

AMERICAN MATHEMATICAL SOCIETY

Volume 348, Number 10, October 1996

\title{
ALGEBRAIC SURFACES WITH LOG CANONICAL SINGULARITIES AND THE FUNDAMENTAL GROUPS OF THEIR SMOOTH PARTS
}

\author{
D.-Q. ZHANG
}

\begin{abstract}
Let $(S, \Delta)$ be a log surface with at worst log canonical singularities and reduced boundary $\Delta$ such that $-\left(K_{S}+\Delta\right)$ is nef and big. We shall prove that $S^{o}=S-\operatorname{Sing} S-\Delta$ either has finite fundamental group or is affine-ruled. Moreover, $\pi_{1}\left(S^{o}\right)$ and the structure of $S$ are determined in some sense when $\Delta=0$.
\end{abstract}

\section{INTRODUCTION}

Let $S$ be a normal projective algebraic surface over the complex number field $\mathbf{C}$ with nef and big anti-canonical divisor $-K_{S}$. Recently, in [11] and [15] (see also $[12,13])$ we have proved that the fundamental group of the smooth part $S^{o}=$ $S-\operatorname{Sing} S$ is finite provided that $S$ has at worst log-terminal singularities (see [5] or [6] for its definition).

In this short note, we shall consider the case when $S$ has at worst log canonical singularities (see (4) of Remark (2.4) below for background and see [4] or [6] for the complete list of log-canonical singularities). Then $\pi_{1}\left(S^{o}\right)$ is no longer finite. However, we shall describe precisely, in the case when $\pi_{1}\left(S^{o}\right)$ is infinite, this fundamental group and the structure of $S$.

The following Lemma (2.1) is rather easy.

Lemma (2.1). Let $S$ be a normal projective algebraic surface with at worst log canonical singularities and with nef and big anti-canonical divisor $-K_{S}$. Suppose that $S$ has at least one non-rational singularity. Then we have:

(1) $S$ is obtained from a nonsingular elliptic ruled surface $\widetilde{S}$ by contracting a cross-section of the ruling to a simple elliptic singularity and several $\mathbf{P}^{1}$ 's in fibers into rational singularities.

(2) We have the following surjective homomorphism, where $S^{o}=S-$ SingS is the smooth part, and hence the infiniteness of $\pi_{1}\left(S^{o}\right)$

$$
\pi_{1}\left(S^{o}\right) \rightarrow \pi_{1}(\widetilde{S})=\mathbf{Z} \times \mathbf{Z} .
$$

The following is one of our main theorems. It says that when $\pi_{1}\left(S^{o}\right)$ is infinite $S$ has a finite Galois covering unramified over $S^{o}$ and with the covering surface described in Lemma (2.1). In Theorems (2.3) and (1.1) below, we refer to [5] or [6]

Received by the editors February 25, 1995 and, in revised form, June 9, 1995.

1991 Mathematics Subject Classification. Primary 14J45; Secondary 14E20, 14J26, 14 J17.

Key words and phrases. Log canonical singularity, nef and big anti-canonical divisor, fundamental group, affine-ruledness. 
for the definition of log canonical singularity and to [4] or [6] for a complete list of log canonical singularities with reduced boundary.

Theorem (2.3). Let $S$ be a normal projective algebraic surface with at worst log canonical singularities and with nef and big anti-canonical divisor $-K_{S}$. Suppose that $S$ has only rational singularities.

Then $S$ is rational, and there is a finite Galois covering $T \rightarrow S$ unramified over $S^{o}:=S-$ SingS, such that $T$ has at worst log canonical singularities and nef and big $-K_{T}$, and that one of the following two cases occurs.

(1) $T^{o}:=T-$ Sing $T$ is simply connected. Hence $T$ is rational and $T$ has at worst rational singularities. Moreover, $\pi_{1}\left(S^{\circ}\right)$ is equal to the finite group $\operatorname{Gal}(T / S)$.

(2) $T$ has exactly one simple elliptic singularity and several rational singularities (see the description of $T$ in Lemma (2.1)). Moreover, $\pi_{1}\left(T^{o}\right)=\mathbf{Z} \times \mathbf{Z}$ and $\pi_{1}\left(S^{o}\right) / \pi_{1}\left(T^{o}\right)=\operatorname{Gal}(T / S)$.

Remark (2.4). (1) Any type of rational singularity which is log canonical but not log terminal can be realized as a singularity on a surface satisfying Case(2) in Theorem (2.3). (See Example (3.1)).

(2) There is no strong relation between the global $\pi_{1}\left(S^{\circ}\right)$ and the local fundamental group at a punctured neighbourhood of a singularity. Indeed, there are examples of surfaces $S$ (cf. Example (3.2)) each of which satisfies Case(1) in Theorem (2.3) and has at least one non-log terminal singularity (with infinite local $\left.\pi_{1}\right)$.

(3) The nef and bigness of $-K_{S}$ implies that the Kodaira dimension $\kappa\left(S,-K_{S}\right)=$ 2. In [17], Sakai denotes $\kappa\left(S,-K_{S}\right)$ by $\kappa^{-1}(S)$. In [12], we consider log terminal surfaces $S$ with $\kappa\left(S,-K_{S}\right)=2$ and try to determine the structure of those $S$ when $S$ - Sing $S$ has infinite fundamental group.

(4) In general, for smooth rational projective surface $X$ with $\kappa^{-1}(X)=2$, Badescu [14] and Sakai [17] have independently proved that $X$ has an anticanonical model $Y$ such that $Y$ is a normal projective surface with at worst rational singularities and ample Q-Cartier divisor $-K_{Y}$.

As pointed out by the referee, $Y$ may have singularities worse than log canonical. However, the category of log canonical singularities is almost the largest one we can handle at the moment. Recall also that Kawamata's canonical model for an open algebraic surface of general type also has at worst log canonical singularities [16]. These are reasons why we assume that $S$ has at worst log canonical singularities.

In Examples (3.1) and (3.2), $S$ has at worst rational log canonical singularities and $-K_{S}$ is ample. One can check that $S$ is nothing but Badescu-Sakai's anticanonical model of the smooth surface $\widetilde{S}$ there.

The above Theorem (2.3) can be reduced to the following Theorem (1.1) by taking a partial resolution of $S$.

Theorem (1.1). Let $S$ be a normal projective surface and let $\Delta$ be a reduced effective divisor such that $(S, \Delta)$ has at worst log canonical singularities. Suppose that $-\left(K_{S}+\Delta\right)$ is nef and big.

Then the open surface $S-$ Sing $S-\Delta$ either has finite fundamental group or is affine-ruled, i.e., it contains $\mathbf{A}^{1} \times C$ for an affine curve $C$.

The crucial results used in the proof of Theorem (1.1) are those in $[8,9]$ on affine-ruledness of open surfaces and in [11] or [12] on fundamental groups of some open rational surfaces. 


\section{Proof of Theorem (1.1)}

Let $(S, \Delta)$ be as in Theorem (1.1). Let $f: \widetilde{S} \rightarrow S$ be a minimal resolution and let $D=\sum_{i=1}^{n} D_{i}$ be the decomposition of the reduced exceptional divisor $D:=f^{-1}(\operatorname{Sing} S)$ into irreducible components. Since $S$ is $\log$ canonical we can write

$$
f^{*}\left(K_{S}+\Delta\right)=K_{\widetilde{S}}+f^{\prime} \Delta+D^{*}
$$

where $D^{*}=\sum_{i} \alpha_{i} D^{*}$ with $0 \leq \alpha_{i} \leq 1$. The graph of each connected component of $D^{*}$, together with local intersection with $f^{\prime} \Delta$, is given in $(1) \sim(10)$ of [6, Fig. 3, pp. 57-58].

Here, if there are nodes in $f^{\prime} \Delta+D^{*}$, we replace $f$ by the composition of the minimal resolution of $S$ and the blowing-up of nodes. Then our $f^{\prime} \Delta+D^{*}$ is simple normal crossing. In other words, we let $f$ be the minimal good resolution of the pair $(S, \Delta)$.

All components in the graphs (4) and (5) of [ibid.], the middle component of the graph (6) or (8) and the middle chains of the graphs (7), (9) and (10) have coefficients one in $D^{*}$, and all the other components of $D$ have coefficients $<1$ in $D^{*}$. Let $g: \widetilde{S} \rightarrow \hat{S}$ be the contraction into a normal surface of all irreducible components of $D$ with coefficient $<1$ in $D^{*}$. Let $\hat{D}=g_{*}(D), \hat{\Delta}=g_{*}\left(f^{\prime} \Delta\right)$.

Lemma (1.2). Let $(S, \Delta)$ be as in Theorem (1.1). Then we have:

(1) The pair $\left(X_{o}:=\hat{S}, B_{o}:=\hat{\Delta}+\hat{D}\right)$ has at worst weaker Kawamata log terminal singularities (wklt, for short) with reduced boundary in the sense of [6] and with nef and big $-\left(K_{X_{o}}+B_{o}\right)$.

(2) $X_{o}$ has at worst log terminal, i.e., quotient singularities.

(3) $S-\operatorname{Sing} S-\Delta=X_{o}-\operatorname{Sing} X_{o}-B_{o}$.

Proof. (1) The log terminality follows immediately from the classification of $\log$ canonical singularities with reduced boundary in [4] or [6]. Since $-\left(K_{S}+\Delta\right)$ is nef and big, so are $-\left(K_{\widetilde{S}}+f^{\prime} \Delta+D^{*}\right)$ and $-\left(K_{\hat{S}}+\hat{\Delta}+\hat{D}\right)$.

(2) Since $\left(X_{o}, B_{o}\right)$ is log terminal in the sense of [6], so is $X_{o}$ by the definition.

(3) is clear from the construction of $(\hat{S}, \hat{\Delta}+\hat{D})$. q.e.d.

We need also the following result. The second assertion of Lemma (1.3) was already proved in Badescu [14] and Sakai [17]. For the reader's convenience, (2) of Lemma (1.3) is also proved here.

Lemma (1.3). Let $(S, \Delta)$ be as in Theorem (1.1). With the above notation, we have:

(1) The minimal good resolution $\widetilde{S}$ of $S$ is a ruled surface such that $\left(f^{\prime} \Delta+\right.$ $\left.\left[D^{*}\right]\right) . F \leq 1$ for a general fiber $F$ of the ruling, where $\left[D^{*}\right]$ is the integral part.

(2) When $\Delta=0, S$ is rational if and only if $S$ has only rational singularities.

Proof. (1) Since $-\left(K_{\widetilde{S}}+f^{\prime} \Delta+D^{*}\right)=-f^{*}\left(K_{S}+\Delta\right)$ is nef and big, $\kappa(\widetilde{S})=-\infty$ and hence $\widetilde{S}$ is ruled. By the Hodge index theorem, $0<-\left(K_{\widetilde{S}}+f^{\prime} \Delta+D^{*}\right) \cdot F=$ $2-\left(f^{\prime} \Delta+D^{*}\right) \leq 2-\left(f^{\prime} \Delta+\left[D^{*}\right]\right)$. The second part of (1) follows.

(2) In view of Lemma (2.1) which will be proved in $\S 2$, we assume that $S$ is an irrational normal surface with at worst rational log canonical singularities and with nef and big $-K_{S}$. We want to get a contradiction. This can be done by the same proof as in [11, Lemma 1.1]. Indeed, by (1), $\widetilde{S}$ is ruled with a ruling $\widetilde{S} \rightarrow C$ onto 
an irrational curve. Since $S$ has at worst rational singularities, $D=f^{-1}(\operatorname{Sing} S)$ is contained in fibers because the base curve has $g(C)=q(\widetilde{S}) \geq 1$. Let $\tau: \widetilde{S} \rightarrow \bar{S}$ be a composition of smooth blowing-downs of curves in fibers into a minimal model. Note that $\tau_{*}\left(D^{*}\right) \sim_{\mathbf{Q}} \alpha \bar{F}$ where $\alpha$ is a non-negative rational number and $\bar{F}$ is a general fiber of the ruling on $\bar{S}$. So we have $0<\left(K_{\widetilde{S}}+D^{*}\right)^{2} \leq\left(K_{\bar{S}}+\tau_{*} D^{*}\right)^{2}=$ $8(1-q(\bar{S}))-4 \alpha \leq 0$. This is a contradiction. This proves Lemma (1.3). q.e.d.

Now we apply the log minimal model programme in [5] for the pair $\left(X_{o}, B_{o}\right)$. Since $K_{X_{o}}+B_{o}$ is not nef, by the cone theorem [5], there are an extremal ray and a corresponding contraction $\sigma_{1}: X_{o} \rightarrow X_{1}$. Note that $\rho\left(X_{1}\right)=\rho\left(X_{o}\right)-1$.

Let $h: \widetilde{X}_{o} \rightarrow X_{o}$ be a minimal resolution. Let $E=\sum_{i=1}^{c} E_{i}$ be the decomposition of the reduced exceptional divisor $E:=h^{-1}\left(\operatorname{Sing} X_{o}\right)$ into irreducible components. Since $\left(X_{o}, B_{o}\right)$ is wklt, we can write

$$
h^{*}\left(K_{X_{o}}+B_{o}\right)=K_{\widetilde{X}_{o}}+h^{\prime} B_{o}+E^{*}
$$

where $E^{*}=\sum_{i} \alpha_{i} D^{*}$ with $0 \leq \alpha_{i}<1[6$, Chapter 3].

Case (1). $\operatorname{dim} X_{1}=0$. Then $\rho\left(X_{o}\right)=1$. Hence $-\left(K_{X_{o}}+B_{o}\right)$ and $-K_{X_{o}}$ are ample. If $B_{o}=0$, then $X_{o}-\operatorname{Sing} X_{o}-B_{o}=S-\operatorname{Sing} S-\Delta$ has finite fundamental group by [11] or [12] or [13] because now $X_{o}$ has at worst log terminal singularities by Lemma (1.2).

Suppose $B_{o} \neq 0$. We shall apply [8, Theorem 2.11] and [9, Main Theorem, Theorem 7 and the Remark after it]. Since $\rho\left(X_{o}\right)=1$ and since $E$ together with its local intersection with $h^{\prime} B_{o}$ is classified in [4] or [6], we see easily, in the notation of [8], that $K_{\widetilde{X}_{o}}+\left(h^{\prime} B_{o}+E\right)^{\#}=K_{\widetilde{X}_{o}}+\left(h^{\prime} B_{o}+E^{*}\right)$ and $\left(\widetilde{X}_{o}, h^{\prime} B_{o}+E\right)$ is almost minimal. Now the results in [8,9] imply that the open surface $\widetilde{X}_{o}-\left(h^{\prime} B_{o}+E\right)=$ $X_{o}-\operatorname{Sing} X_{o}-B_{o}=S-\operatorname{Sing} S-\Delta$ has finite fundamental group or is affine-ruled.

We note that in [9], $X_{o}$ is assumed to be rational. However, if $X_{o}$ is not rational, we shall see that $X_{o}-\operatorname{Sing} X_{o}-B_{o}$ is affine-ruled. In the present case, $\widetilde{X}_{o}$ is an irrational ruled surface by Lemma (1.3). Let $F$ be a general fiber of the ruling. By Lemma (1.2) (2), $E$ consists of $\mathbf{P}^{1}$ 's and hence $E=h^{-1}\left(\operatorname{Sing} X_{o}\right)$ is contained in fibers because the base curve of the ruling has genus $>0$. Thus $F \cdot\left(h^{*} B_{o}+E\right)=$ $F . h^{\prime} B_{o} \leq 1$ by Lemma (1.3). So, $X_{o}-\operatorname{Sing} X_{o}-B_{o}=\widetilde{X}_{o}-E-h^{\prime} B_{o}$ contains an $\mathbf{A}^{1}$ - or $\mathbf{P}^{1}$-fibration with a general fiber $F-h^{\prime} B_{o}$, and hence it is affine-ruled.

Case (2). $\operatorname{dim} X_{1}=1$. We shall prove that $X_{o}-\operatorname{Sing} X_{o}-B_{o}=S-\operatorname{Sing} S-\Delta$ is affine-ruled. Let $F$ be a general fiber of the fibration $\sigma_{1}: X_{o} \rightarrow X_{1}$. Then $F \cong \mathbf{P}^{1}\left[5\right.$, Remark 5-1-1]. Note that $F \cdot B_{o}=h^{*} F \cdot h^{\prime} B_{o} \leq 1$ by Lemma (1.3). So, $X_{o}-\operatorname{Sing} X_{o}-B_{o}$ contains an $\mathbf{A}^{1}$ - or $\mathbf{P}^{1}$-fibration with a general fiber $F-B_{o}$, whence it is affine-ruled.

Case (3). $\operatorname{dim} X_{1}=2$. We first prove the following:

Lemma (1.4). Suppose Case (3) occurs. Then we have:

(1) $\left(X_{1}, B_{1}:=\sigma_{1 *} B_{o}\right)$ is wklt with reduced boundary $B_{1}$ and with nef and big $-\left(K_{X_{1}}+B_{1}\right)$.

(2) If $X_{1}-\operatorname{Sing} X_{1}-B_{1}$ is affine-ruled or has finite fundamental group, then so is $X_{o}-\operatorname{Sing} X_{o}-B_{o}=S-\operatorname{Sing} S-\Delta$, respectively.

Proof. (1) comes from [5, Proposition 5-1-6] and the corresponding fact for $\left(X_{o}, B_{o}\right)$. 
(2) This follows from the following equality

$$
\begin{gathered}
X_{1}-\text { Sing } X_{1}-B_{1}-(\text { one smooth point if necessary }) \\
=X_{o}-\operatorname{Sing} X_{o}-B_{o}-\left(\sigma_{1}-\text { exceptional divisor }\right)
\end{gathered}
$$

and hence the surjectivity of the natural homomorphism (cf. e.g. [10]):

$$
\pi_{1}\left(X_{1}-\operatorname{Sing} X_{1}-B_{1}\right) \rightarrow \pi_{1}\left(X_{o}-\operatorname{Sing} X_{o}-B_{o}\right)=\pi_{1}(S-\operatorname{Sing} S-\Delta) .
$$

This proves Lemma 1.4. q.e.d.

If Case (1) or (2) takes place, then Theorem (1.1) is proved. If Case (3) above is true we apply Lemma (1.4) above and the same argument above for $\left(X_{1}, B_{1}\right)$. In Case (3), $1 \leq \rho\left(X_{1}\right)=\rho\left(X_{o}\right)-1$, so Case (3) can only occur for finitely many times. Namely, there is a composition of contractions of extremal rays $\sigma: \hat{S}=X_{o} \rightarrow X$ such that the pair $\left(X, B:=\sigma_{*} B_{o}\right)$ is wklt with reduced boundary $B$, with nef and big $-\left(K_{X}+B\right)$ and satisfying Case (1) or (2) above. Now the affine-ruledness or the finiteness of the fundamental group of $X-\operatorname{Sing} X-B$ proved in Cases (1) and (2) above implies the same result for $X_{o}-\operatorname{Sing} X_{o}-B_{o}=S-\operatorname{Sing} S-\Delta$ by Lemma (1.4). So, Theorem (1.1) is true.

This completes the proof of Theorem (1.1). $\quad$ q.e.d.

\section{Proof of Theorem (2.3)}

We first prove Lemma (2.1) which is stated in the Introduction.

Let $S$ be as in Lemma (2.1). We employ the following notations with $\Delta=0$ used at the beginning of $\S 1: f: \widetilde{S} \rightarrow S, D:=\sum_{i=1}^{n} D_{i}=f^{-1}(\operatorname{Sing} S), D^{*}=\sum_{i} \alpha_{i} D_{i}$ and

$$
f^{*}\left(K_{S}\right)=K_{\widetilde{S}}+D^{*}
$$

We may assume that $\sum_{i=1}^{r} D_{i}$ is a connected component of $D$ which is mapped to a non-rational singularity on $S$ (the existence of such a singularity is guaranteed by the assumption in Lemma (2.1)). So, $f\left(\sum_{i=1}^{r} D_{i}\right)$ is a simple elliptic singularity, or a cusp, and $\sum_{i=1}^{r} D_{i}$ is an elliptic curve or a simple loop of $\mathbf{P}^{1}$ 's. Moreover, $\alpha_{i}=1$ for $i=1, \cdots, r[6$, Chapter 2].

By Lemma (1.3), $\widetilde{S}$ is a ruled surface with $\left[D^{*}\right] . F \leq 1$. Here $F$ is a general fiber of the ruling $\widetilde{S} \rightarrow C$.

If $\widetilde{S}$ is rational, then $\left|K_{\widetilde{S}}+D^{*}\right| \supseteq\left|K_{\widetilde{S}}+D_{1}+\cdots+D_{r}\right| \neq \phi[7$, Lemma 2.1.3, p. 7], a contradiction. Thus, $\widetilde{S}$ is an irrational ruled surface and $g(C)=q(\widetilde{S}) \geq 1$.

Note that $\sum_{i=1}^{r} D_{i}$ contains at least one component, say $D_{1}$, vertical to the ruling on $\widetilde{S}$. Thus, $r=1,\left[D^{*}\right] \cdot F=D_{1} \cdot F=1$ and $D_{1}$ is a nonsingular elliptic curve. So $g(C)=1$ and $\widetilde{S}$ is elliptic ruled.

Now $F$. $\left(\left[D^{*}\right]-D_{1}\right)=0$. So $\left[D^{*}\right]-D_{1}$ is contained in fibers of $\widetilde{S} \rightarrow C$. Hence all singularities other than $f\left(D_{1}\right)$ are rational (cf. [6, Fig. 3, pp. $\left.57-58\right]$ ). So, $D-D_{1}$ consists of $\mathbf{P}^{1}$ 's and is contained in fibers because the base curve $C$ is irrational.

We have proved Lemma (2.1) (1). The surjectivity in Lemma (2.1) (2) is now clear because $S^{o}=\widetilde{S}-D$ (cf. e.g. [10]). $\quad$ q.e.d.

Next we shall prove the following :

Theorem (2.2). Let $S$ be a normal projective surface with at worst log canonical singularities and with nef and big anti-canonical divisor $-K_{S}$. Suppose that $S$ has 
only rational singularities and that the fundamental group $\pi_{1}\left(S^{\circ}\right)$ of the smooth part $S^{\circ}=S-$ Sing $S$ is infinite. Then we have:

(1) There is a finite Galois covering $T \rightarrow S$ unramified over $S^{\circ}$ such that $T$ has at worst log canonical singularities and nef and big $-K_{T}$. Moreover $T$ has exactly one simple elliptic singularity and several rational singularities (cf. Lemma (2.1)).

(2) We have $\pi_{1}\left(T^{o}\right)=\mathbf{Z} \times \mathbf{Z}$ where $T^{o}=T-\operatorname{Sing} T$, and $\pi_{1}\left(S^{o}\right) / \pi_{1}\left(T^{o}\right)=$ $\operatorname{Gal}(T / S)$.

Proof. Let $S$ be as in Theorem (2.2). We employ the following notations with $\Delta=0$ used at the beginning of $\S 1$ :

$$
f: \widetilde{S} \rightarrow S, \quad D=f^{-1}(\operatorname{Sing} S)
$$

Let $S^{o}=S-\operatorname{Sing} S$. Then $S^{o}=\widetilde{S}-D=\hat{S}-\operatorname{Sing} \hat{S}-\hat{D}$ (see the notation for the latter set in $\S 1$ ). Now by Lemma (1.2) and Theorem (1.1), $S^{o}$ is affine-ruled because of the infiniteness assumption on $\pi_{1}\left(S^{\circ}\right)$. So, we have a rational map $\xi: \widetilde{S} \rightarrow B$ to a complete smooth curve $B$ such that a general fiber of the restriction map $\xi_{\mid S^{\circ}}$ contains $\mathbf{A}^{1}$.

By Lemma (1.3), $\widetilde{S}$ is rational and hence $B=\mathbf{P}^{1}$. Let $Z \rightarrow \widetilde{S}$ be a composition of blowing-ups such that $\xi$ induces a morphism $\eta: Z \rightarrow B$. Let $D_{Z}$ be the inverse on $Z$ of $D$.

First we have

$$
\pi_{1}\left(S^{o}\right)=\pi_{1}\left(Z-D_{Z}\right)
$$

because the blowing-up of smooth points on $S$ does not affect $\pi_{1}\left(S^{o}\right)$. Secondly, a general fiber $F$ of $\eta$ is $\mathbf{P}^{1}$ and a general fiber $F^{o}=F-D_{Z}$ of $\eta_{\mid Z-D_{Z}}$ contains $\mathbf{A}^{1}$. Hence $D_{Z} \cdot F \leq 1$ and $F^{o}=\mathbf{A}^{1}$ or $\mathbf{P}^{1}$.

Since $D_{Z}$ is contractible to the singular locus on $S, D_{Z}$ contains no full fiber of $\eta$. So, every fiber of the fibration $\eta_{\mid Z-D_{Z}}$ is 1-dimensional, and we let $F_{1}^{o}, \cdots, F_{r}^{o}$ be all fibers of this fibration with $m_{i}:=$ (g.c.d. of coefficients of irreducible components in $\left.F_{i}^{o}\right)>1$.

If $r \leq 2$, then $B-\sum_{i} \eta\left(F_{i}^{o}\right)=\mathbf{C}^{*}, \mathbf{A}^{1}$ or $\mathbf{P}^{1}$ and the proof of [10, Lemma 1.5] guarantees the existence of the following exact sequence:

$$
(1)=\pi_{1}\left(F^{o}\right) \rightarrow \pi_{1}\left(Z-D_{Z}\right) \rightarrow \pi_{1}\left(B-\sum_{i} \eta\left(F_{i}^{o}\right)\right) .
$$

Hence $\pi_{1}\left(S^{o}\right)=\pi_{1}\left(Z-D_{Z}\right)$ is abelian. Thus $\pi_{1}\left(S^{o}\right)=H_{1}\left(S^{o}, \mathbf{Z}\right)$. The latter is finite because $S$ is rational and by considering the cohomology exact sequence of the pair $(\widetilde{S}, D)$ (using also Poincaré duality) [11, Lemma 1.3]. So $\pi_{1}\left(S^{o}\right)$ is finite. This contradicts the assumption in Theorem (2.2).

Thus $r \geq 3$. By the solution of [1, 2] to Fenchel's conjecture, there exists a Galois covering from a complete smooth curve $C$ onto $B$ such that it is ramified exactly over $\eta\left(F_{i}^{o}\right)$ and the ramification index of each point lying over $\eta\left(F_{i}^{o}\right)$ is equal to $m_{i}$. Let $\widetilde{T} \rightarrow Z$ be the normalization of $Z \times{ }_{B} C$.

The natural Galois covering $\widetilde{T} \rightarrow Z$ is unramified over $Z-D_{Z}$ by the construction. So, if we let $T \rightarrow S$ be the normalization of $S$ in the function field of $\mathbf{C}(\widetilde{T})$, then $T \rightarrow S$ is unramified over $S^{o}$. Note that $T$ also has at worst log canonical singularities (cf. the proof of [3, Proposition 1.7]) and nef and big $-K_{T}$ which is the pull back of $-K_{S}$. Moreover, $\pi_{1}\left(S^{o}\right) / \pi_{1}\left(T^{o}\right)=\operatorname{Gal}(T / S)$ where $T^{o}=T-\operatorname{Sing} T$. 
Let $D_{\widetilde{T}}$ be the inverse on $\widetilde{T}$ of $D_{Z}$. We have also

$$
\pi_{1}\left(T^{o}\right)=\pi_{1}\left(\widetilde{T}-D_{\widetilde{T}}\right)
$$

because there is a composition of smooth blowing-downs

$$
\widetilde{T}-D_{\widetilde{T}} \longrightarrow T^{o}-\text { (several smooth points if necessary). }
$$

Note that $D_{\widetilde{T}} \cdot F_{\widetilde{T}} \leq 1$ where $F_{\widetilde{T}}$ is a general fiber of the $\mathbf{P}^{1}$-fibration $\widetilde{T} \rightarrow C$ induced from the $\mathbf{P}^{1}$-fibration $\eta: Z \rightarrow B$. Hence a general fiber $F_{\widetilde{T}}^{o}=F_{\widetilde{T}}-D_{\widetilde{T}}$ of the surjective restriction morphism $\widetilde{T}-D_{\widetilde{T}} \rightarrow C$ is $\mathbf{A}^{1}$ or $\mathbf{P}^{1}$. The g.c.d. of the coefficients of irreducible components in each fiber of this restriction morphism is one by the construction of the covering. Applying the proof of [10, Lemma 1.5] again, we get an exact sequence:

$$
(1)=\pi_{1}\left(F_{\widetilde{T}}^{o}\right) \rightarrow \pi_{1}\left(\widetilde{T}-D_{\widetilde{T}}\right) \rightarrow \pi_{1}(C) \rightarrow(1) .
$$

Hence we have:

$$
\pi_{1}\left(\widetilde{T}-D_{\widetilde{T}}\right)=\pi_{1}(C), \pi_{1}\left(Z-D_{Z}\right) / \pi_{1}\left(\widetilde{T}-D_{\widetilde{T}}\right)=\operatorname{Gal}(\widetilde{T} / Z)
$$

By the infiniteness assumption on $\pi_{1}\left(S^{o}\right)=\pi_{1}\left(Z-D_{Z}\right)$ in Theorem $(2.2), \pi_{1}(C)$ is infinite. Hence (the irregularity of a smooth model of $\widetilde{T}$ ) $=g(C) \geq 1$, and $C$ and $\widetilde{T}$ are irrational. By Lemma (1.3), $T$ has an irrational singularity and hence $T$ is as described in Lemma (2.1). In particular, $g(C)=1$ and $C$ is a nonsingular elliptic curve. Thus by the results in the previous paragraph, we have:

$$
\pi_{1}\left(T^{o}\right)=\pi_{1}\left(\widetilde{T}-D_{\widetilde{T}}\right)=\mathbf{Z} \times \mathbf{Z}, \pi_{1}\left(S^{o}\right) / \pi_{1}\left(T^{o}\right)=\operatorname{Gal}(T / S) .
$$

This completes the proof of Theorem (2.2). q.e.d.

We now prove Theorem (2.3) which is stated in the Introduction. By Lemma (1.3), $S$ is rational. If $\pi_{1}\left(S^{o}\right)$ is infinite, then Theorem (2.3) follows from Theorem (2.2) and Lemma (2.1). So we may assume $\pi_{1}\left(S^{o}\right)$ is finite. We extend the universal covering of $S^{o}$ to a Galois covering $T \rightarrow S$ unramified over $S^{o}$. By the proof of [3, Proposition 1.7], $T$ has at worst $\log$ canonical singularities and nef and big $-K_{T}$ which is the pull back of $-K_{S}$. Since $T^{o}=T-\operatorname{Sing} T$ is simply connected, $T$ has only rational singularities by Lemma (2.1). Thus, $T$ is rational by Lemma (1.3). So, the assertions in Theorem (2.3) are all true.

Theorem (2.3) is proved. q.e.d.

\section{Proof of Remark (2.4)}

The list of rational singularities which are log canonical but not log terminal, is given in [4] or [6]. To be precise, these are singularities of types (6), (7) and (8) of [6, Fig. 3, pp. 57-58]; (7) and (8) are essentially of the same type.

In Example (3.1) below we shall give examples of singularity type (8) and all three subtypes $(3,3,3),(2,4,4),(2,3,6)$ of $(6)$ such that the surfaces fit Case (2) in Theorem (2.3). In Example (3.2), we shall give examples of singularity type (7) such that the surfaces fit Case (1) in Theorem (2.3).

Example (3.1). Let $\pi: \mathbf{P}^{1} \times \mathbf{P}^{1} \rightarrow \mathbf{P}^{1}$ be the projection to the first component. Let $M$ be a section with $M^{2}=0$. Let $\sigma: \widetilde{S} \rightarrow \mathbf{P}^{1} \times \mathbf{P}^{1}$ be a composition of blowing-ups of 3 or 4 points on $M$ and several infinitely near points of them, such that $M^{\prime}=\sigma^{\prime} M$ and all (3 or 4) singular fibers of the $\mathbf{P}^{1}$-fibration $\widetilde{S} \rightarrow \mathbf{P}^{1}$, induced from $\pi$, are given in one of Figures 1,2,3 and 4. Let $f: \widetilde{S} \rightarrow S$ be the contraction of $M^{\prime}$ and all components in singular fibers except the $(-1)$-curve in each singular 


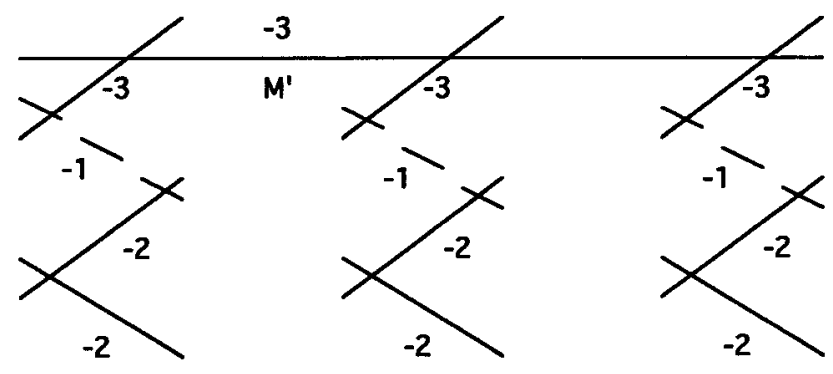

Figure 1

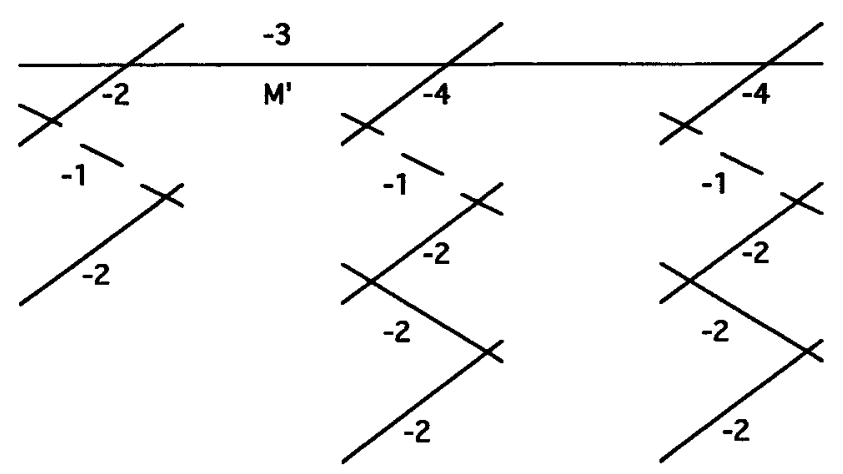

Figure 2

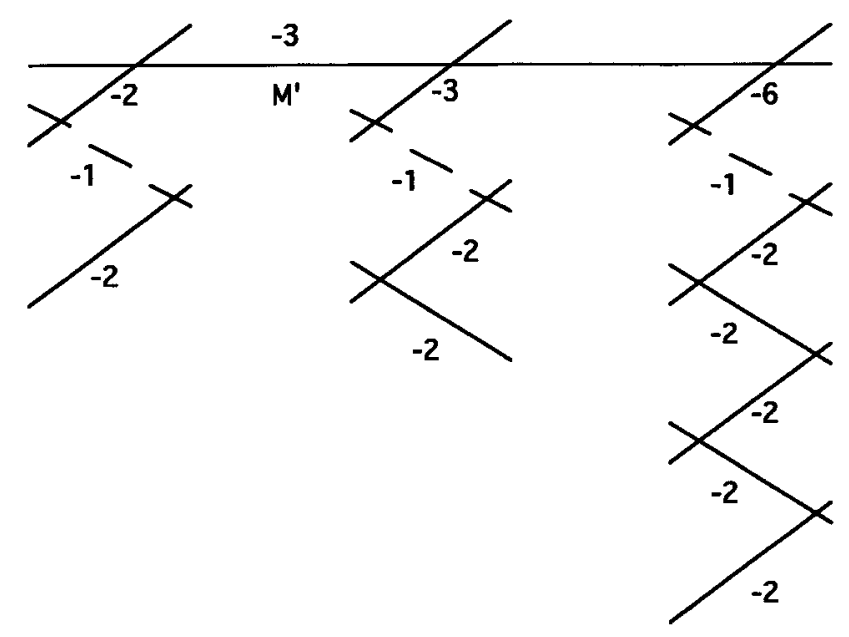

Figure 3 


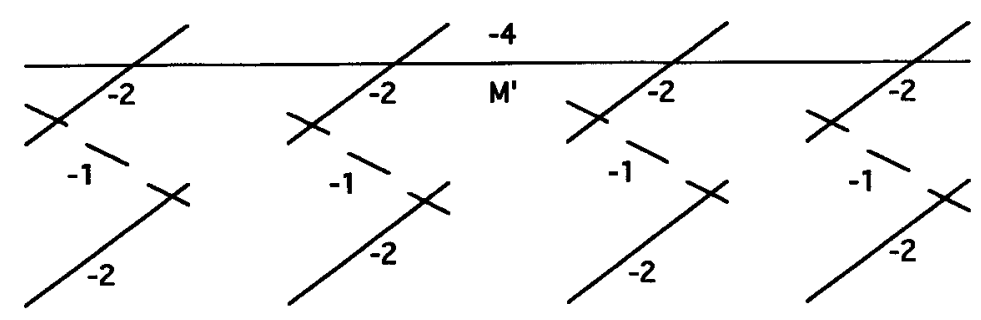

Figure 4

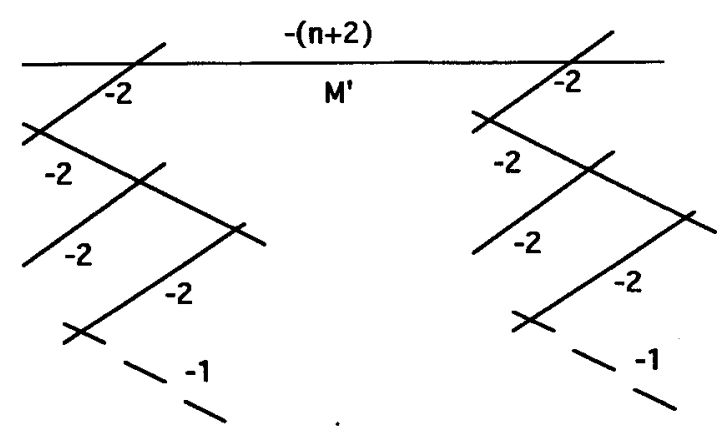

FiguRE 5

fiber. Then we see that the connected component of the $f$-exceptional divisor containing $M^{\prime}$ is contracted to a rational singularity of either one of subtypes $(3,3,3),(2,4,4)$ and $(2,3,6)$ in (6), or type (8) in [ibid.]. All the other singularities of $S$ are cyclic quotient, hence $\log$ terminal and also $\log$ canonical. So, $S$ has at worst rational log canonical singularities.

We see easily that $\rho(S)=1$ and $-K_{S}$ is ample. Moreover, $S$ with Figure (1), (2), (3) or (4) fits Case (2) of Theorem (2.3) with $\operatorname{Gal}(T / S)=\mathbf{Z} / 3 \mathbf{Z}, \mathbf{Z} / 4 \mathbf{Z}, \mathbf{Z} / 6 \mathbf{Z}$ or $\mathbf{Z} / 2 \mathbf{Z}$, respectively. Here $T$ is a smooth elliptic ruled surface with $e$-invariant equal to $6,8,12$ or 4 , respectively.

Example (3.2). Let $\pi: \Sigma_{n} \rightarrow \mathbf{P}^{1}$ be the ruling on the Hirzebruch surface $\Sigma_{n}$ of degree $n(n \geq 1)$. Let $M$ be the minimal section on $\Sigma_{n}$ with $M^{2}=-n$. Let $\sigma: \widetilde{S} \rightarrow \Sigma_{n}$ be a composition of blowing-ups of 2 points on $M$ and 6 infinitely near points of them, such that $M^{\prime}=\sigma^{\prime} M$ and all two singular fibers of the $\mathbf{P}^{1}$-fibration $\widetilde{S} \rightarrow \mathbf{P}^{1}$, induced from $\pi$, are given in Figure 5 . Let $f: \widetilde{S} \rightarrow S$ be the contraction of $M^{\prime}$ and all components in singular fibers except the $(-1)$-curve in each singular fiber. Then we see that the $f$-exceptional divisor is connected and contracted to a rational singularity of type (7) in [ibid.]. Hence $S$ has at worst rational log canonical singularities.

We see easily that $\rho(S)=1$ and $-K_{S}$ is ample. This $S$ fits Case(1) of Theorem (2.3) with $\operatorname{Gal}(T / S)=\mathbf{Z} / 2 \mathbf{Z}$. Here $T$ is a rational surface with $\rho(T)=3$ and with exactly one singularity. Moreover, the singularity on $T$ is also of type (7) in [ibid.]. 


\section{REFERENCES}

1. T. C. Chau, A note concerning Fox's paper on Fenchel's conjecture, Proc. Amer. Math. Soc. 88 (1983), 584-586. MR 84m:20038

2. R. H. Fox, On Fenchel's conjecture about F-groups, Math. Tidsskr. B. (1952), 61-65. MR 14:843c

3. Y. Kawamata, The cone of curves of algebraic varieties, Ann. of Math. 119 (1984), 603-633. MR 86c: $14013 b$

4. Y. Kawamata, The crepant blowing-up of 3-dimensional canonical singularities and its application to degenerations of surfaces, Ann. of Math. 127 (1988), 93-163. MR 89d:14023

5. Y. Kawamata, K. Matsuda and K. Matsuki, Introduction to the minimal model problem, Adv. Study Pure Math. 10 (1987), 283-360. MR 89e:14015

6. J. Kollár, Flip and abundance for algebraic threefolds, Astérisque 211 (1992). MR 94f:14013

7. M. Miyanishi, Non-complete algebraic surfaces, Lecture Notes in Mathematics, vol. 857, Springer-Verlag, 1981. MR 83b:14011

8. M. Miyanishi and S. Tsunoda, Non-complete algbraic surfaces with logarithmic Kodaira dimension $-\infty$ and with non-connected boundaries at infinity, Japan. J. Math. 10 (1984), 195-242. MR 88b:14029

9. M. Miyanishi and S. Tsunoda, Logarithmic del Pezzo surfaces of rank one with noncontractible boundaries, Japan. J. Math. 10 (1984), 271-319. MR 88b:14030

10. M. V. Nori, Zariski's conjecture and related problems, Ann. Sci. Éc. Norm. Sup. 16 (1983), 305-344. MR 86j:14027

11. D.-Q. Zhang, Algebraic surfaces with nef and big anti-canonical divisor, Math. Proc. Cambridge Philos. Soc. 117 (1995), 161-163. MR 95:02

12. D.-Q. Zhang, Normal algebraic surfaces of anti-Kodaira dimension one or two, Intern. J. Math. 6 (1995), 329-336.

13. A. Fujiki, R. Kobayashi and S. Lu, On the fundamental group of certain open normal surfaces, Saitama Math. J. 11 (1993), 15-20. MR 94m:32042

14. L. Badescu, Anticanonical models of ruled surfaces, Ann. Univ. Ferrara 29 (1983), 165-177. MR 85m: 14056

15. R. V. Gurjar and D.-Q. Zhang, $\pi_{1}$ of smooth points of a log del Pezzo surface is finite: I, II, J. Math. Sci. Univ. Tokyo 1 (1994), 137-180; 2 (1995) 165-196. MR 95m:14015; CMP 95:17

16. Y. Kawamata, On the classification of non-complete algebraic surfaces, Lecture Notes in Mathematics, vol. 732, Springer-Verlag, 1979, pp. 215-232. MR 81c:14021

17. F. Sakai, Anticanonical models of rational surfaces, Math. Ann. 269 (1984), 389-410. MR 85m:14058

18. D.-Q. Zhang, The fundamental group of the smooth part of a log Fano variety, Osaka J. Math. 32 (1995), 637-644. CMP 96:06

Department of Mathematics, National University of Singapore, Singapore

E-mail address: matzdq@nus.sg 